\title{
Injectable peptide hydrogels for controlled drug release
}

Morgane Mannes ${ }^{1}$, Charlotte Martin ${ }^{1}$, Maria Dumitrascuta ${ }^{2}$, Yannick Van Wanseele ${ }^{3}$, Sophie Hernot ${ }^{4}$, Ann Van Eeckhaut ${ }^{3}$, Annemieke Madder ${ }^{5}$, Richard Hoogenboom ${ }^{6}$, Mariana Spetea ${ }^{2}$, Steven Ballet ${ }^{1}$

\author{
${ }^{1}$ Research Group of Organic Chemistry, Vrije Universiteit Brussel, Belgium \\ ${ }^{2}$ Department of Pharmaceutical Chemistry, University of Innsbruck, Austria \\ ${ }^{3}$ Department of Pharmaceutical Chemistry and Drug Analysis, Vrije Universiteit Brussel, Belgium \\ ${ }^{4}$ In Vivo Cellular and Molecular Imaging, Vrije Universiteit Brussel, Belgium \\ ${ }^{5}$ Organic and Biomimetic Chemistry Research Group, Ghent University, Krijgslaan, 281, 9000 Ghent, Belgium \\ ${ }^{6}$ Supramolecular Chemistry Group, Ghent University, Krijgslaan 281, 9000 Ghent, Belgium
}

https://doi.org/10.17952/35EPS.2018.286

Chronic pain remains one of the main challenges in human medicine at the beginning of the third millennium, with ca. $20-30 \%$ people worldwide suffering from chronic pain.[1] One of the major medical priorities is to provide effective pain control and sustained analgesia. Here, the focus was placed on the use of peptide-based hydrogels as a controlled drug release platform. Hydrogels are three-dimensional cross-linked networks able to retain large amounts of water. The porous structure of the gel allows the encapsulation of active compounds and their slow release from the hydrogel network in order to maintain a therapeutic concentration of the drug in the systemic circulation over an extended period (Figure 1).

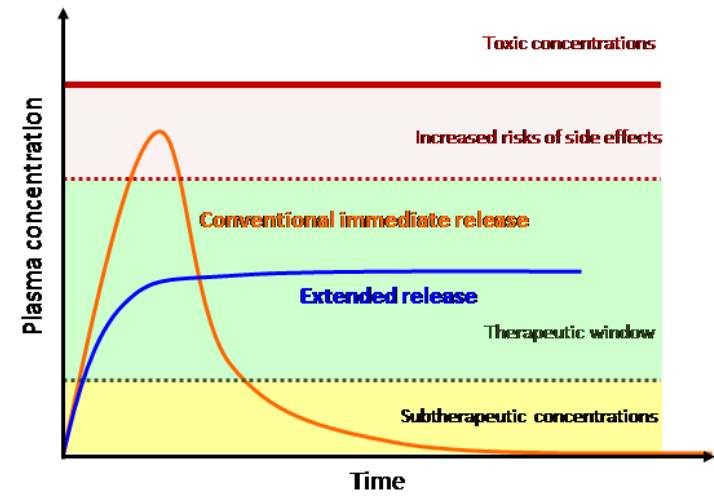

Figure 1: Schematic representation of plasma concentrations in function of time following administration of immediate-release and extended-release formulations. [2]

In case of pain therapy based on opioids, oscillating drug blood levels are avoided, reducing the frequency of administration and drug dosage, and resulting in less "clock-w atching" and a better night time pain control.[2] The extended drug release, herein provided by the hydrogels, present a reduced risk of toxicity and side effects. Additionally, due to encapsulation into the matrix, the use of hydrogels protects the drug from premature in vivo degradation, increasing the drug's half-life.
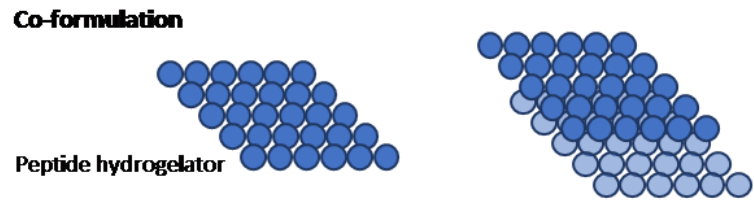

$\beta$-strand

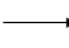

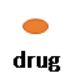

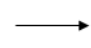

Biogel formulation
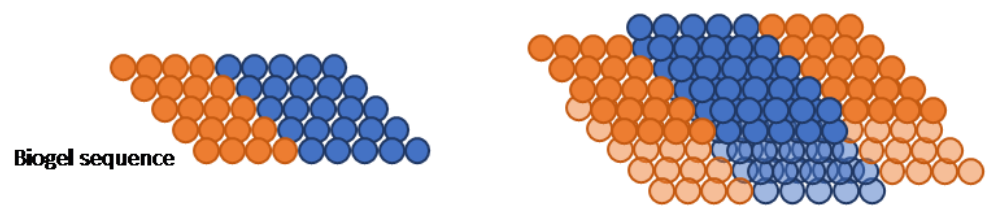

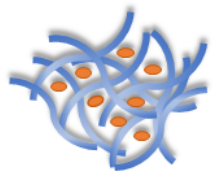

Bioactive hydrogel network

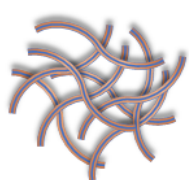

Figure 2: Schematic representation of the self-assembly process of hydrogels based on amphipathic peptide sequences. Both the 'co-formulation' and 'biogel' strategies are depicted.[5] 
The focus was placed on amphipathic peptide-based hydrogels, because of their biocompatibility and biodegradability. The peptidic hydrogelator sequences are build-up of alternating hydrophobic and hydrophilic amino acids and have the tendency to self-assemble in aqueous media to form a hydrogel network.[3]

In this work, the peptide hydrogelators are short, but still tunable, sequences, enabling a plethora of possible modifications in view of the optimization of gelation and drug release properties. The injectability of these hydrogels allows an administration through parenteral delivery, including intramuscular and subcutaneous injection.[4]

In order to provide sustained analgesia with adequate efficacy, the focus was placed on the development of improved opioid-based therapies. To obtain the desired analgesic activity with reduced side effects over extended periods of time, two different strategies were exploited, as depicted in Figure 2. Either the opioid pharmacophore is encapsulated or co-formulated into the hydrogel network, or the peptidic opioid is part of the hydrogel sequence, giving rise to an analgesic gel, herein called biogel.[5] The peptide opioid pharmacophores were designed based on endogenous opioid peptides, and were linked with previously developed lead hydrogelators.[6.7]

The hydrogel-encapsulated peptide opioids and the biogel conjugates were tested in vivo, to determine their extended antinociceptive effect (Figure 3). The biogels, were compared to their corresponding parent opioid sequences co-formulated with a hydrogelator. A similar profile and strength were obtained for both strategies, even though the biogel required higher doses to achieve an equianalgesic effect. This variation in dosage is explained by a difference in release mechanism of the drug. While for the co-formulation the drug is released by diffusion through the hydrogel network, for the biogel an enzymatic degradation of the biogel is necessary to release the opioid pharmacophore and induce the observed analgesic effect.[5]

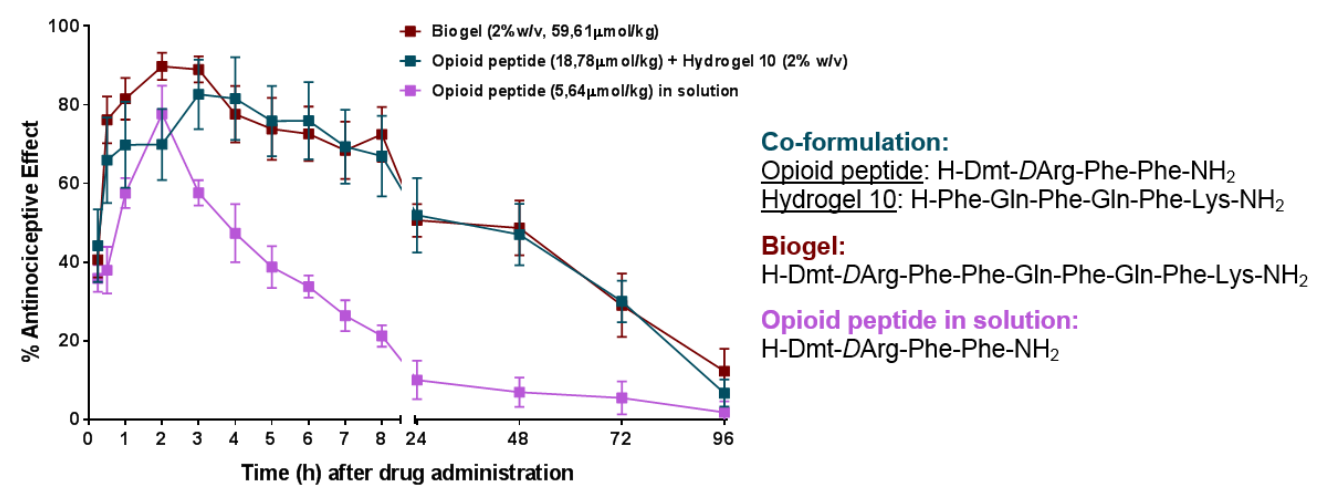

Figure 3: Comparison of antinociceptive activities of the opioid peptide co-formulated with Hydrogel 10 or conjugated to Hydrogel 10, resulting in the 'biogel', in the tail-flick test after s.c. administration in mice. [5]

We demonstrated that by using both strategies an extended antinociceptive effect up to 72 to $96 \mathrm{~h}$ was induced after subcutaneous injection in mice.[5] These promising results open up a research avenue for improved chronic pain therapies, but it is expected that the presented formulations are also more broadly applicable for other biologically active peptide therapeutics.

\section{References}

[1] Kapur, B. M.; Lala, P. K.; Shaw, J. L., Pharmacogenetics of chronic pain management. Clinical Biochemistry 2014, 47 (13), 1169-1187.

[2] Martin, C.; De Baerdemaeker, A.; Poelaert, J.; Madder, A.; Hoogenboom, R.; Ballet, S., Controlled-release of opioids for improved pain management. Materials Today 2016, 19 (9), 491-502.

[3] Dasgupta, A.; Mondal, J. H.; Das, D., Peptide hydrogels. RSC Advances 2013, 3 (24), 9117-9149.

[4] Bibian, M.; Mangelschots, J.; Gardiner, J.; Waddington, L.; Acevedo, M.M.D.; De Geest, B.G.; Van Mele, B.; Madder, A.; Hoogenboom, R.; Ballet, S., Rational design of a hexapeptide hydrogelator for controlled-release drug delivery. Journal of Materials Chemistry B 2015, 3 (5), 759-765.

[5] Martin, C.; Dumitrascuta, M.; Mannes, M.; Lantero, A.; Bucher, D.; Walker, K.; Van Wanseele, Y.; Oyen, 
E.; Hernot, S.; Van Eeckhaut, A.; Madder, A.; Hoogenboom, R.; Spetea, M.; Ballet, S., Journal of Medicinal Chemistry 2018 DOI: 10.1021/acs.jmedchem.8b01282.

[6] Martin, C.; Oyen, E.; Mangelschots, J.; Bibian, M.; Ben Haddou, T.; Andrade, J.; Gardiner, J.; Van Mele, B.; Madder, A.; Hoogenboom, R.; Spetea, M.; Ballet, S., Injectable peptide hydrogels for controlled-release of opioids. MedChemComm 2016, 7, 542-549.

[7] Martin, C.; Oyen, E.; Van Wanseele, Y.; Haddou, T. B.; Schmidhammer, H.; Andrade, J.; Waddington, L.; Van Eeckhaut, A.; Van Mele, B.; Gardiner, J., Injectable peptide-based hydrogel formulations for the extended in vivo release of opioids. Materials Today Chemistry 2017, 3, 49-59. 